\title{
Laser wakefield simulation using a speed-of-light frame envelope model
}

\author{
B. Cowan*, D. Bruhwiler*, E. Cormier-Michel ${ }^{\dagger}$, E. Esarey ${ }^{\dagger, * *}$, \\ C. G. R. Geddes ${ }^{\dagger}$, P. Messmer* and K. Paul* \\ *Tech-X Corporation, 5621 Arapahoe Ave., Boulder, CO 80303 \\ ${ }^{\dagger}$ LOASIS program, Lawrence Berkeley National Laboratory, Berkeley, CA 94720 \\ ** University of Nevada, Reno, Reno, NV 89557
}

\begin{abstract}
Simulation of laser wakefield accelerator (LWFA) experiments is computationally intensive due to the disparate length scales involved. Current experiments extend hundreds of laser wavelengths transversely and many thousands in the propagation direction, making explicit PIC simulations enormously expensive and requiring massively parallel execution in 3D. We can substantially improve the performance of laser wakefield simulations by modeling the envelope modulation of the laser field rather than the field itself. This allows for much coarser grids, since we need only resolve the plasma wavelength and not the laser wavelength, and therefore larger timesteps. Thus an envelope model can result in savings of several orders of magnitude in computational resources. By propagating the laser envelope in a frame moving at the speed of light, dispersive errors can be avoided and simulations over long distances become possible. Here we describe the model and its implementation, and show simulations and benchmarking of laser wakefield phenomena such as channel propagation, self-focusing, wakefield generation, and downramp injection using the model.
\end{abstract}

\section{INTRODUCTION}

Laser-driven plasma wakefield accelerators (LWFAs) are capable of producing accelerating gradients several orders of magnitude higher than conventional accelerators. Several years ago, high-quality electron beams were produced by self-trapping and accelerated to $\sim 100 \mathrm{MeV}$ in a few millimeters $[1,2,3]$. More recently, acceleration to $1 \mathrm{GeV}$ in a few centimeters was demonstrated [4]. In addition, experiments have shown that the quality of the electron beam can be improved by controlling the injection process separately from the accelerating structure [5, 6]. Having successfully proven the principle of laser-plasma acceleration, an important goal is to extend LWFAs toward the capabilities of a high-energy collider. This involves achieving $\sim 10 \mathrm{GeV}$ of energy gain in single meter-scale acceleration stages, and further optimizing the injection process to reduce emittance.

Simulations have played a significant role in all of these developments. In particular, the particle-in-cell (PIC) method, a well-established algorithm for self-consistently modeling charged particles in electromagnetic fields [7, 8], has been critical to the understanding of the LWFA process. However, future explorations present difficulties for PIC simulations, due to the disparate length scales involved. The laser wavelength typically used is $\lambda=800 \mathrm{~nm}$; this must be well-resolved in a PIC simulation, and because of the Courant limit [9], the time step must then be a small fraction of a laser oscillation period. At the same time, meter-scale acceleration stage designs use a lower plasma density for 
a longer dephasing length, and correspondingly longer laser pulses. Thus the simulation must encompass many thousands of cells longitudinally and hundreds transversely, and run for tens of millions of timesteps. This is intractable even in two dimensions. Also, running PIC simulations of the injection process in 3D is expensive enough that it makes parameter studies for beam quality optimization quite difficult. New tools are therefore needed to perform these simulations more quickly while preserving as much as possible of the physics modeled by PIC.

In order to overcome the disparities in length scale, an algorithm was proposed which models not the fast laser oscillation itself, but the slowly-varying envelope modulating the fast sinusoidal oscillation [10]. Removing the need to resolve the laser wavelength allows for larger grid spacings and thus larger timesteps as well. The algorithm was implemented in the plasma simulation code VORPAL [11] and tested in limited cases [12]. More recently, an improvement was made to the algorithm in order to resolve problems which appeared with long propagation distances [13]. In this article, we explore this improved algorithm using its implementation in VORPAL. First, we describe the algorithm. Then we present some benchmark tests of the envelope model against explicit PIC. Finally, we show some examples of LWFA simulations which are attainable using the model.

\section{DESCRIPTION OF THE ALGORITHM}

As the envelope algorithm is described in detail in $[10,13,12]$, we only summarize it here. The laser envelope model consists of the electromagnetic fields and particles of standard PIC along with a complex scalar field $a$ which represents the envelope modulating the oscillation of the transverse vector potential of the laser field. Specifically, the transverse vector potential in the Coulomb gauge is given in terms of $a$ by

$$
\mathbf{A}_{\perp}(t, \mathbf{x})=\operatorname{Re}\left[\hat{\boldsymbol{\epsilon}} a(t, \mathbf{x}) e^{i\left(\omega t-k_{0} x\right)}\right]
$$

where $\omega$ is the angular frequency of the laser, $k_{0}=\omega / c$ is the free-space wavenumber, and $\hat{\boldsymbol{\epsilon}}$ is a constant polarization vector with $|\hat{\boldsymbol{\epsilon}}|^{2}=1$. The PIC electromagnetic fields then represent only the fields due to the plasma dynamics, and not the laser field.

The envelope model update has several steps which are modified from that of standard PIC. First, the particles are adapted to include the effects of the ponderomotive force from the laser field. The particle model tracks the momentum $\overline{\mathbf{p}}$ averaged over a laser oscillation period and also the average relativistic factor which accounts for the quiver motion

$$
\bar{\gamma}=\sqrt{1+\frac{|\overline{\mathbf{p}}|^{2}}{m^{2} c^{2}}+\frac{q^{2}|a|^{2}}{2 m^{2} c^{2}}},
$$

where $m$ is the particle mass and $q$ is the charge. The particle update then includes the ponderomotive force, with the momentum equation

$$
\frac{d \overline{\mathbf{p}}}{d t}=q(\mathbf{E}+\mathbf{v} \times \mathbf{B})-\frac{q^{2}}{4 \bar{\gamma} m} \boldsymbol{\nabla}|a|^{2} .
$$


Here $\mathbf{E}$ and $\mathbf{B}$ are the PIC electromagnetic fields, and the average velocity $\mathbf{v}=\overline{\mathbf{p}} / \bar{\gamma} m$ is used. The numerical procedure for the paricle push is described in [10]. The electromagnetic fields are updated and the particles deposited as in standard PIC.

The laser envelope field is updated separately from the PIC electromagnetic fields. The envelope update is performed in a Galilean frame co-moving with the laser field at the speed of light, with coordinates given by $(\tau=t, \xi=x-c t)$. Applying the Maxwell equations to the laser field in the speed-of-light frame yields the approximate update equation

$$
\left[\frac{2}{c} \frac{\partial}{\partial \tau}\left(\frac{\partial}{\partial \xi}-i k_{0}\right)+\nabla_{\perp}^{2}\right] a=-\mu_{0} \chi a .
$$

Here $\chi$ is the plasma susceptibility, which is derived from the particles by

$$
\chi=-\sum_{j} \frac{q_{j} \rho_{j}}{\bar{\gamma}_{j} m},
$$

where $q_{j}, \rho_{j}$, and $\bar{\gamma}_{j}$ are the charge, charge density distribution, and average relativistic factor, respectively, for the $j$ th particle. The numerical procedure for the envelope field update remains the same as in [12], but with the light-frame update equation substituted for the lab-frame equation.

\section{BENCHMARK TESTS}

Linear propagation in a plasma channel. In order to test the envelope model, we examine the case of propagation of a low-intensity laser pulse with a transverse profile matched to a plasma channel. We know that a Gaussian laser pulse with waist parameter $w_{0}$ is matched to a parabolic plasma channel with radial profile $n(r)=n_{0}+r^{2} / \pi r_{e} w_{0}^{4}$, where $r_{e}=e^{2} / 4 \pi \epsilon_{0} m c^{2}$ is the classical electron radius [14]. The eigenmodes of the channel then vary longitudinally as $e^{-i k_{x} x}$, and obey the dispersion relation $k^{2}=k_{x}^{2}+k_{1}^{2}$, where $k=\omega / c$ for that mode and we define the parameter $k_{1}$ by

$$
k_{1}^{2}=4\left(\frac{1}{w_{0}^{2}}+\pi r_{e} n_{0}\right) .
$$

The group velocity $v_{g}$ is then given by

$$
\beta_{g}=\frac{v_{g}}{c}=\left(1+\frac{k_{1}^{2}}{k_{x}^{2}}\right)^{-1 / 2} .
$$

Now the envelope update equation is an approximation to the Maxwell equations, and yields the dispersion relation $k=k_{x}+k_{1}^{2} / 2 k_{x}$, which gives $\beta_{g}=1-k_{1}^{2} / 2 k_{x}$. We see that both these expressions agree with the full Maxwell relations to first order in $k_{1} / k_{x}$, which should be acceptable since for long accelerator stages which employ relatively low densities of $n_{0} \sim 10^{23} \mathrm{~m}^{-3}, k_{1} \ll k_{0}$.

To test the dispersion characteristics of the envelope model, we choose parameters deliberately to exacerbate any dispersion errors that might occur, namely, we set $n_{0}=$ 

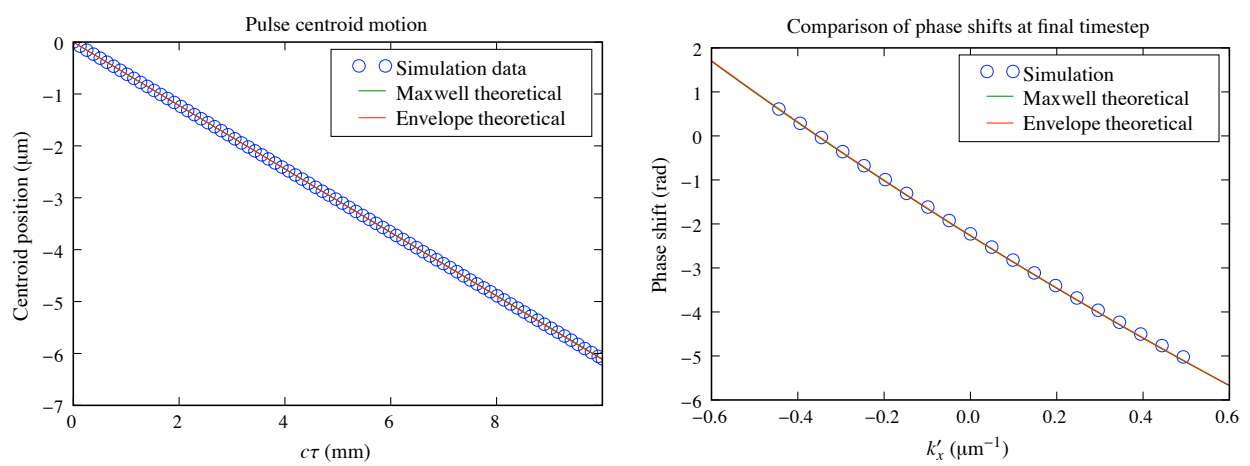

FIGURE 1. Left: The centroid position of the envelope field. Right: The phase of the spatial frequency components.

$10^{24} \mathrm{~m}^{-3}$ and $w_{0}=10 \mu \mathrm{m}$, so that both the background plasma density and the narrow channel contribute to the dispersion. We run in $3 \mathrm{D}$ and propagate for $1 \mathrm{~cm}$, then examine the dispersion characteristics quantitatively. The left plot in Fig. 1 shows the centroid positions of the envelope at each timestep as well as the theoretical values from the Maxwell and envelope group velocities. We can see the pulse walk backward in the speed-of-light frame due to the sub-luminal group velocity. In the right plot, we use a Fourier transform to decompose the envelope into spatial frequency components, and compare the phases of each against the theoretical values derived from the dispersion relations; here $k_{x}^{\prime}=k_{x}-k_{0}$. In both comparisons, the Maxwell and envelope theoretical values are nearly identical, and both agree well with the simulation results.

$1 D$ wakefield tests. The wakefield is a critical quantity for LWFA simulations. In order to test the reliability of the wakefields produced by the envelope model, we compare the model to explicit PIC for a set of one-dimensional simulations. We choose to work in 1D because explicit PIC does not experience grid dispersion in that case, which would otherwise cause a group velocity error. We set our parameters for a wide separation in length scale: a laser RMS pulse length of $32 \mu \mathrm{m}$, with a matched plasma wavelength of $201 \mu \mathrm{m}$ for a density of $2.76 \times 10^{22} \mathrm{~m}^{-3}$. For the explicit simulation, we choose a relatively high resolution of 64 grid cells per wavelength to minimize discretization errors [15], and for the envelope model we use 40 cells per plasma wavelength. The results are shown in Fig. 2. We see that the envelope model differs in amplitude by a relatively small amount, and the phase difference is a small fraction of a plasma wavelength, even for $a_{0}=3.0$. In that case, the amplitude difference was $1.0 \%$, and the phase difference was $120 \mathrm{mrad}$. The agreement between the envelope and explicit simulations was much better than when the explicit simulations were run with just 24 cells per wavelength, indicating that the envelope model does not experience the same errors as explicit PIC.

The parameters used in these tests are comparable to those for a $20 \mathrm{GeV}$ accelerating stage with $a_{0}=1$, and the speedup gained from the envelope model is $\sim 10^{4}$. Such a large speedup is not unexpected, as at lower densities, the plasma wavelength and matched 

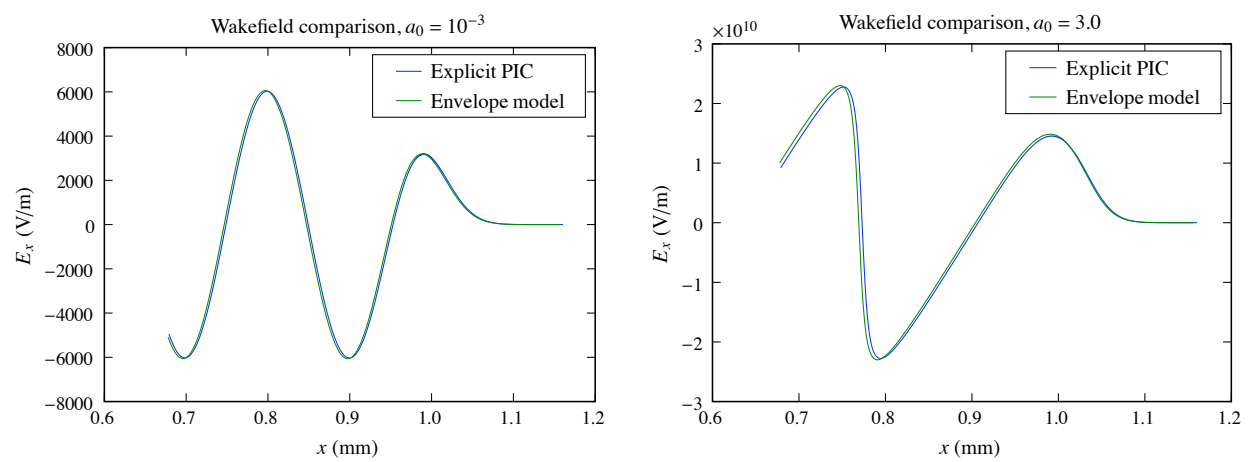

FIGURE 2. Comparisons between explicit PIC and the envelope model in 1D.

laser pulse length increase. Since these are the smallest scales that need to be resolved, the grid spacing and timestep can both be increased as well. Speedup in 2D and 3D is similar, since the transverse resolution in explicit PIC need not be as high as the longitudinal resolution.

\section{APPLICATIONS OF THE ENVELOPE MODEL}

We now show examples of the application of the envelope model in the two areas needing more efficient simulations: long-distance propagation and injection. The left plot of Fig. 3 shows the fluence profiles of a laser field propagating in a plasma channel relevant for a $10 \mathrm{GeV}$ stage. We use a plasma density of $10^{23} \mathrm{~m}^{-3}$ at the bottom of the channel and $a_{0}=1$. The self-focusing oscillations are clearly visible. The simulation propagated the laser pulse for $55 \mathrm{~cm}$ in $360 \mathrm{CPU}$ hours in 2D. The envelope model was also used with scaled parameters (see [16]) for comparison with explicit PIC. In those tests, good agreement was observed and a speedup factor of 18 was obtained. The propagation distance is limited by the spectral broadening that the laser pulse experiences as it depletes. Ultimately, the broadened pulse becomes unresolved by the larger grid spacing that the envelope model allows.

The right plot of Fig. 3 shows the phase space of the electrons in a simulation of downramp injection using the envelope model, obtaining similar results to [5]. This demonstrates that the envelope model can describe particle trapping despite the reduced representation of the laser field - a task that the more severe quasi-static approximation does not allow.

\section{CONCLUSION}

The laser envelope model is an exciting tool for LWFA simulation. Its ability to reduce the required computational time by removing the need to resolve the laser wavelength allows it to reach new application areas, while still agreeing with both theory and 

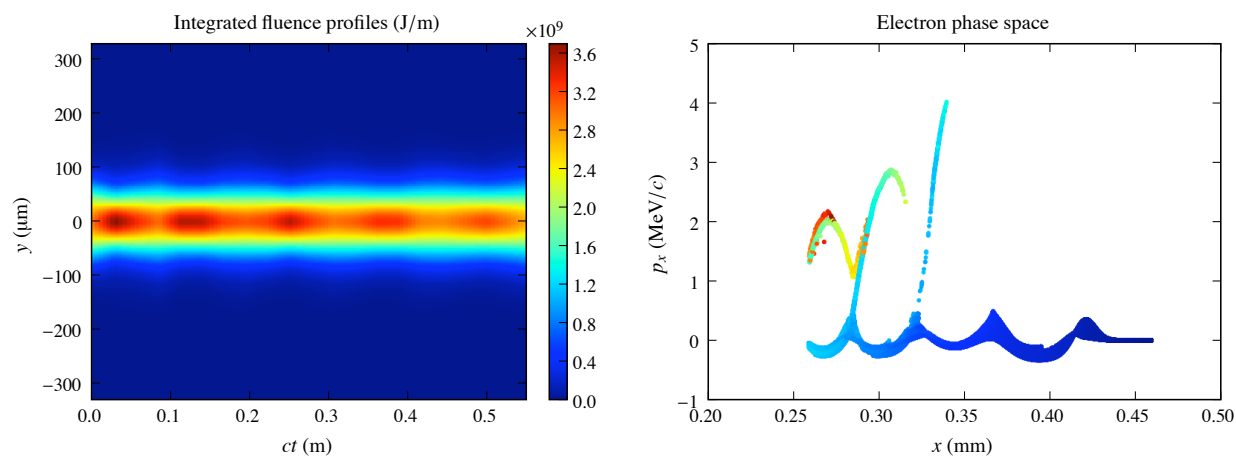

FIGURE 3. Left: Transverse profiles of a laser pulse propagating in a plasma channel, showing selffocusing oscillations. Right: Longitudinal phase space in a downramp injection simulation.

explicit PIC in simple benchmark tests. Future work includes further benchmarking and exploration of LWFA parameter spaces for meter-scale acceleration and downramp injection.

\section{ACKNOWLEDGMENTS}

The authors would like to acknowledge the assistance of the VORPAL development team. Work supported by Department of Energy contracts DE-AC02-05CH11231 (LBNL), DE-FC02-07ER41499 (SciDAC), and DE-FG02-04ER84097 (SBIR).

\section{REFERENCES}

1. C. G. R. Geddes et al., Nature 431, 538 (2004).

2. S. P. D. Mangles et al., Nature 431, 535 (2004).

3. J. Faure et al., Nature 431, 541 (2004).

4. W. P. Leemans et al., Nature Physics 2, 696-699 (2006).

5. C. G. R. Geddes et al., Phys. Rev. Lett. 100, 215004 (2008).

6. A. Gonsalves et al., these proceedings (2008).

7. C. K. Birdsall and A. B. Langdon, Plasma Physics via Computer Simulation, McGraw-Hill, New York, NY, 1985.

8. R. W. Hockney and J. W. Eastwood, Computer Simulation Using Particles, McGraw-Hill, New York, NY, 1981.

9. A. Taflove and S. C. Hagness, Computational Electrodynamics: The Finite-Difference Time-Domain Method, Artech House, Boston, 2000, second edn.

10. D. Gordon, W. Mori, and T. Antonsen, Jr., IEEE Trans. Plasma Science 28, 1135-1143 (2000).

11. C. Nieter and J. R. Cary, J. Comp. Phys 196, 538 (2004).

12. P. Messmer and D. L. Bruhwiler, Phys. Rev. ST Accel. Beams 9, 031302 (2006).

13. D. Gordon, IEEE Trans. Plasma Science 35, 1486-1488 (2007).

14. E. Esarey and W. P. Leemans, Phys. Rev. E 59, 1082-1095 (1999).

15. E. Cormier-Michel et al., Physical Review E (Statistical, Nonlinear, and Soft Matter Physics) 78, 016404 (2008).

16. E. Cormier-Michel et al., "Scaled simulations of a $10 \mathrm{GeV}$ accelerator," in these proceedings, 2008. 